\title{
Facile hydrothermal synthesis of flower-like hematite microstructure with high photocatalytic properties
}

\author{
Timur Sh. ATABAEV* \\ Department of Physics and Astronomy, Seoul National University, Gwanak-gu, Seoul 151-747, Republic of Korea
}

Received: July 09, 2014; Revised: October 26, 2014; Accepted: October 27, 2014

(C) The Author(s) 2015. This article is published with open access at Springerlink.com

\begin{abstract}
A flower-like hematite microstructure has been successfully prepared by a facile hydrothermal synthesis method without using any organic solvents or templates. It is revealed that the flower-like hematite microstructure consists of well-crystallized nanorods with the average diameter of about $100 \pm 15 \mathrm{~nm}$ and average length of about $900 \pm 100 \mathrm{~nm}$ growing from the centers. A possible growth mechanism of the flower-like $\alpha-\mathrm{Fe}_{2} \mathrm{O}_{3}$ microstructure is proposed and discussed. The photocatalytic properties of the synthesized flower-like hematite nanostructure are evaluated using the degradation of rhodamine $B$ in aqueous solution.
\end{abstract}

Keywords: hematite; nanostructure; hydrothermal synthesis

\section{Introduction}

Due to their low cost and environmental friendliness, hematite or $\alpha-\mathrm{Fe}_{2} \mathrm{O}_{3}$ has been the subject of intense research for several decades. Hematite nanostructures are extensively studied for solar water splitting $[1,2]$, new lithium battery materials [3], water treatment [4], etc. Over the past few years, various hematite nanostructures, such as nanoparticles [5], nanowires [6] and nanotubes [7] have been prepared with a variety of methods. These methods demonstrate that morphological control of hematite can be achieved by using different solvents. However, these common organic-phase methods are hazardous because these precursors are toxic and difficult to handle under normal conditions. Moreover, these approaches are unsuitable in terms of scalling up for industrial production owing to the high cost and large volume of chemical wastes generated [8].

* Corresponding author.

E-mail: atabaev@snu.ac.kr, timuratabaev@yahoo.com
Three-dimensional (3D) hierarchical architectures which are constructed from one-dimensional and two-dimensional nanoscaled building blocks have aroused extensive attention owing to their enhanced physical/chemical properties and superior device performances [9]. Such hierarchical architectures combine the features of nanoscaled building units and can exhibit unique properties that are distinct from those of the low-dimensional structures. Therefore, researchers continue to seek straightforward and inexpensive chemical methods for controllable production of 3D hierarchical functional nanostructures. Thus, it remains a significant challenge to develop a simple, effective and template-free method to construct complex 3D hierarchical structures at relatively low temperatures.

Herein, novel 3D flower-like hematite microstructure was prepared via a simple hydrothermal method without using any templates at a relatively low temperature. The structure and morphology of the resulting hematite microstructures were characterized by X-ray diffraction (XRD), field emission scanning 
electron microscopy (FESEM), transmission electron microscopy (TEM) and energy-dispersive X-ray spectroscopy (EDX). The photocatalytic properties of synthesized 3D flower-like hematite were investigated using the degradation of rhodamine $\mathrm{B}$ in aqueous solution. The present study reveals a fast and reliable approach for building hierarchically self-assembled hematite microstructures.

\section{Experimental}

Analytical grade $\mathrm{FeCl}_{3}$ (97.0\%) and $\mathrm{NaNO}_{3}$ (99.0\%) were purchased from Sigma-Aldrich Corporation and were used without further purification. In a typical synthesis method, $\mathrm{FeCl}_{3}(0.015 \mathrm{~mol})$ and $\mathrm{NaNO}_{3}$ $(0.1 \mathrm{~mol})$ were dissolved in $40 \mathrm{~mL}$ of deionized (DI) water. Precipitates were prepared by heating the aqueous solution of corresponding metal salts in sealed glass bottle at $100{ }^{\circ} \mathrm{C}$ for $2 \mathrm{~h}$. The resulting colloidal solution was centrifuged at $5000 \mathrm{rpm}$ for $10 \mathrm{~min}$. The precipitates were washed 3 times with ethanol and DI water, dried and calcined in air at $700{ }^{\circ} \mathrm{C}$ for $1 \mathrm{~h}$.

The structure of the prepared powders was examined by XRD (Bruker D8 Discover) using $\mathrm{Cu} \mathrm{K} \alpha$ radiation $(\lambda=0.15405 \mathrm{~nm})$ at $2 \theta$ scan range of $20^{\circ}-60^{\circ}$. The morphology of the particles was characterized by FESEM (Hitachi S-4700) and TEM (JEOL JEM-2100F). Elemental analysis was carried out using EDX (Horiba, 6853-H). The thermogravimetric (TG) analysis was performed with a Netzsch-STA-449C differential scanning calorimetry-thermogravimetric analyzer in air atmosphere from room temperature up to $700{ }^{\circ} \mathrm{C}$ at elevating temperature rate of $10{ }^{\circ} \mathrm{C} / \mathrm{min}$. The prepared hematite microstructure was further used as a photocatalyst for the rhodamine $\mathrm{B}(\mathrm{RB})$ aqueous solution degradation. In a typical process, $50 \mathrm{mg}$ of the flower-like hematite sample was added to $100 \mathrm{~mL}$ of $1.0 \times 10^{-5} \mathrm{M}$ RB solution and then magnetically stirred in the dark for $30 \mathrm{~min}$, which made the adsorption of $\mathrm{RB}$ on the flower-like sample reach equilibrium. The solution was then exposed to ultraviolet (UV) irradiation with a distance of $20 \mathrm{~cm}$ from a $250 \mathrm{~W}$ high-pressure $\mathrm{Hg}$ lamp at room temperature. Every $5 \mathrm{~min}, 5 \mathrm{~mL}$ of solution was withdrawn from the beaker and then centrifuged. The concentrations of RB in the supernatants were analyzed using a UV-Vis spectrophotometer (Evolution 220) at a wavelength of $553 \mathrm{~nm}$. All measurements were performed at room temperature.

\section{Results and discussion}

The morphology and elemental analysis of the prepared flower-like hematite microstructure were examined by FESEM and EDX. As shown in Fig. 1, the sample is composed of uniform flower-like architectures with the diameter of $3-5 \mu \mathrm{m}$. The magnified image of individual flower-like architecture (inset of Fig. 1) reveals that each structure is composed of many aligned nanorods with the average diameter of about $100 \pm 10 \mathrm{~nm}$ and average length of about $900 \pm$ $100 \mathrm{~nm}$ growing from the center. EDX analysis of the structure reveals only the presence of $\mathrm{Fe}$ and $\mathrm{O}$ in the formed microstructure ( $\mathrm{Pt}$ peaks appear due to platinum coating on the sample). This indicates that the material is clean, and no sodium, neither nitrate or chloride contamination, is detected which indicates a great improvement compared to solvothermal or template-based synthesis. It is also found that the reaction time and $\mathrm{FeCl}_{3}$ concentration have no significant effects on the morphology of products (Fig. S1 in the Electronic Supplementary Material (ESM)). However, a minimum time of $2 \mathrm{~h}$ is needed to prepare the flower-like $\mathrm{FeOOH}$ microstructure. Thus, one can

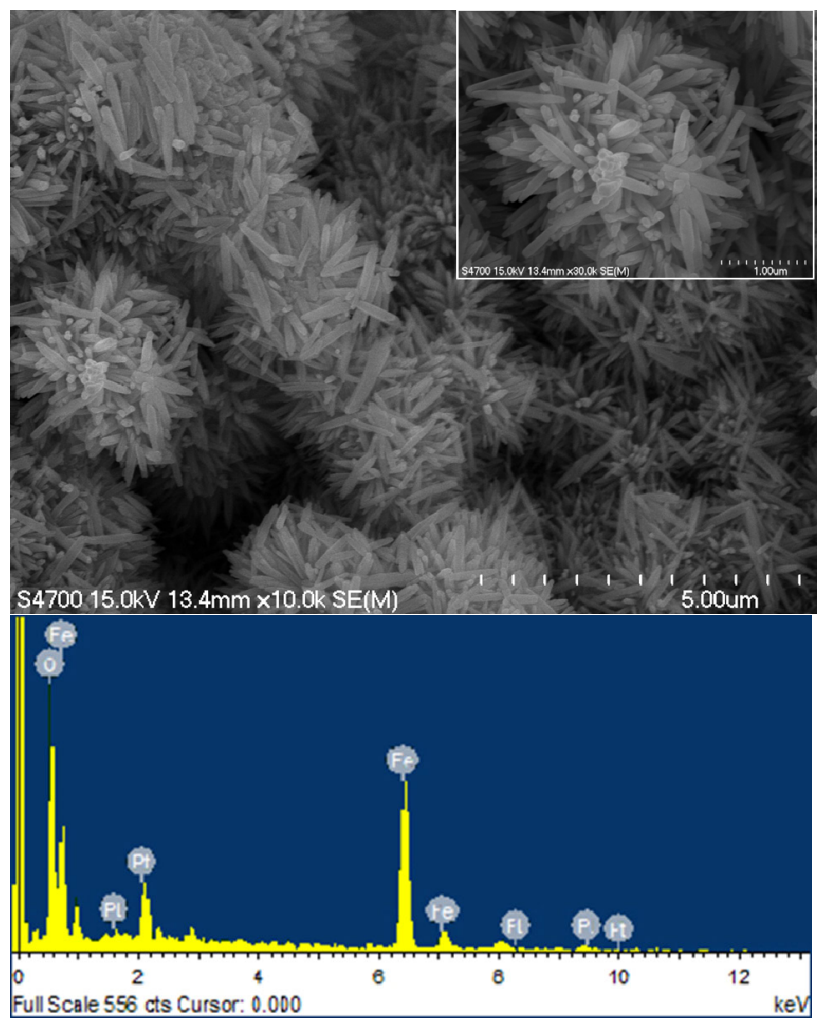

Fig. 1 FESEM and EDX analysis of the flower-like hematite microstructure. 
synthesize the flower-like microstructure within a relatively short time frame. TG analysis (Fig. S2 in the ESM) shows that the total mass loss corresponding to the complete transformation of $\mathrm{FeOOH}$ into $\alpha-\mathrm{Fe}_{2} \mathrm{O}_{3}$ should be around $\sim 20 \%$, which is fully achieved at $700{ }^{\circ} \mathrm{C}$. Further temperature rising probably leads to a strong agglomeration and is not investigated in the present study.

The microstructure of the as-prepared flower-like $\alpha-\mathrm{Fe}_{2} \mathrm{O}_{3}$ sample is further examined by TEM. Figure 2(a) displays the representative TEM image of the obtained $\alpha-\mathrm{Fe}_{2} \mathrm{O}_{3}$ flower-like pattern. TEM micrograph clearly indicates that the sample is built up with radially aligned nanorods, which is consistent with the observation from the FESEM image. The high resolution TEM image (inset of Fig. 2(a)) of the selected area marked with a white rectangle shows a crystalline character with a lattice spacing of $0.248 \mathrm{~nm}$, which is close to the interplanar distance for the (110) plane of $\alpha-\mathrm{Fe}_{2} \mathrm{O}_{3}$. Figure 2(b) displays the typical XRD pattern of the flower-like $\alpha-\mathrm{Fe}_{2} \mathrm{O}_{3}$ sample, and all the peaks can be indexed to the rhombohedral phase of
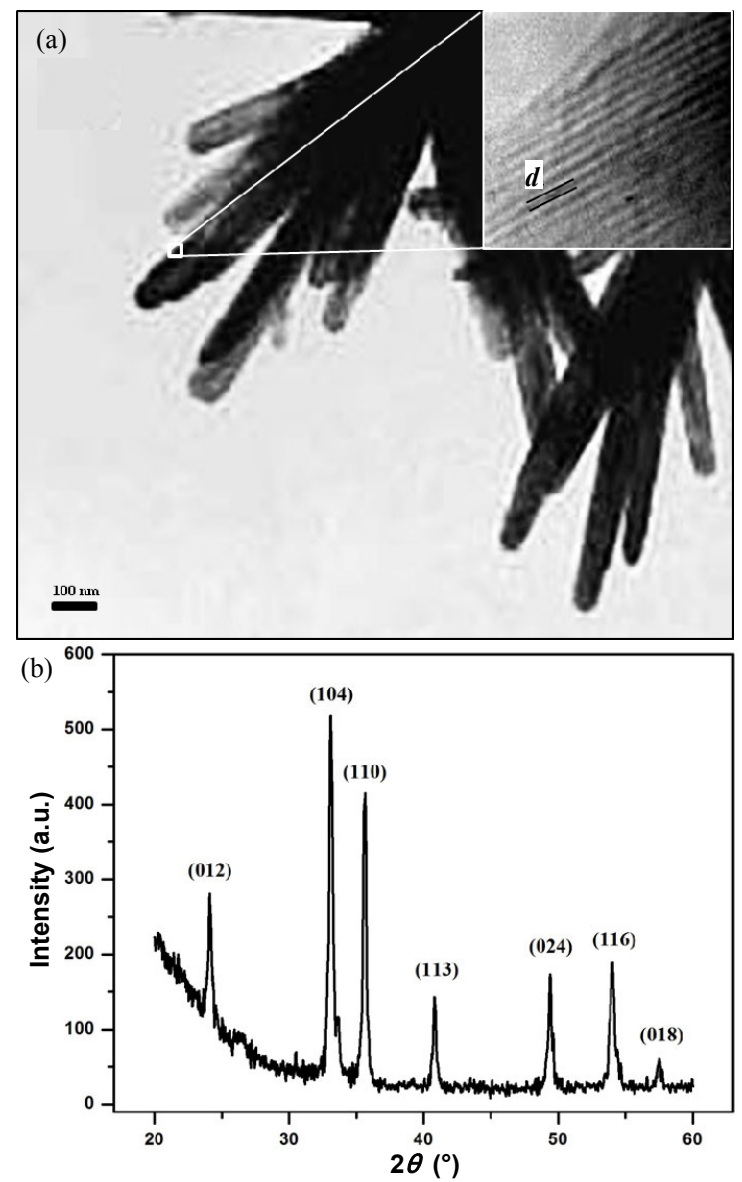

Fig. 2 (a) TEM and (b) XRD analysis of the flower-like hematite microstructure.
$\alpha-\mathrm{Fe}_{2} \mathrm{O}_{3}$ (JCPDS No. 33-0664). The narrow and sharp peaks suggest that the obtained $\alpha-\mathrm{Fe}_{2} \mathrm{O}_{3}$ is highly crystalline. The crystallite size of the $\alpha-\mathrm{Fe}_{2} \mathrm{O}_{3}$ sample evaluated by Scherrer's equation for the strongest peak (104) is $27.3 \mathrm{~nm}$, suggesting that nanorods are consisted of smaller crystallites.

The possible growth mechanism of the flower-like $\alpha-\mathrm{Fe}_{2} \mathrm{O}_{3}$ is schematically shown in Fig. 3. It is well known that the kinetics of $\mathrm{Fe}^{3+}$ hydrolysis in aqueous solutions and the morphologies and properties of solid hydrolytic products strongly depend on the nature of the anion involved. Therefore, the use of $\mathrm{NaNO}_{3}$ is crucial in the experiment. At the first stage, iron(III)-oxyhydroxide particles are produced by forced hydrolisis of iron(III) chloride. With the heat-treatment proceeding, the particles become more spherical, since a spherical shape is optimal from an energetic point of view. As the heat-treated time prolongs, the crystals continue to grow by combining with the remaining primary particles, probably due to Ostwald ripening. Finally, nanorods form, grow and merge with each other, forming the flower-like structure.

The photocatalytic performance of the flower-like $\alpha-\mathrm{Fe}_{2} \mathrm{O}_{3}$ sample is investigated by monitoring the rhodamine $\mathrm{B}(\mathrm{RB})$ aqueous solution degradation under UV irradiation. For comparative purposes, the photodegradation efficiencies of RB mediated by commercially available hematite powders and without any photocatalyst under UV irradiation are also investigated. The changes in the concentration of RB with UV irradiation time are plotted and shown in Fig. 4. In the absence of any catalyst, the concentration of $\mathrm{RB}$ decreases gradually and about $63 \%$ of $\mathrm{RB}$ is degraded under UV irradiation within $60 \mathrm{~min}$. The commercial hematite powders show very weak photocatalytic activity under UV irradiation $(71 \%$ of

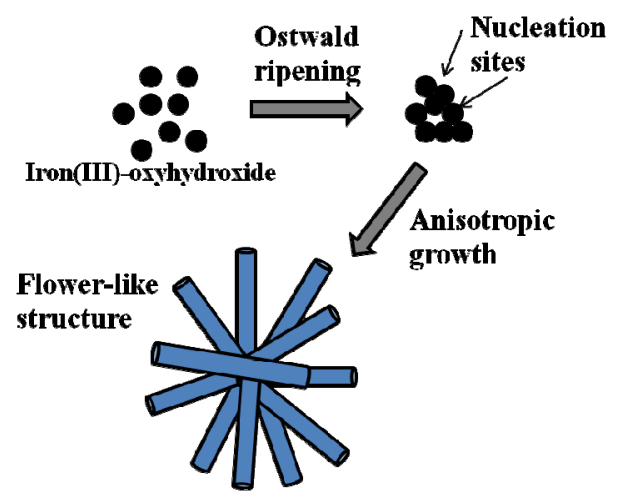

Fig. 3 Possible formation mechanism of the flower-like hematite microstructure. 


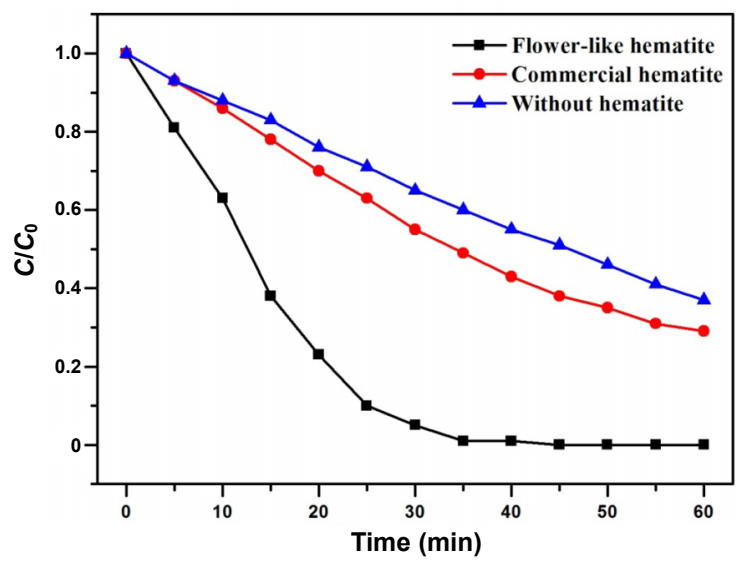

Fig. 4 Photocatalytic performances of the flower-like hematite and commercial hematite powders at different time under exposure to UV irradiation $(C$ : the concentration of RB at designated time; $C_{0}$ : the initial concentration of RB).

$\mathrm{RB}$ within $60 \mathrm{~min}$ ). However, the concentration of $\mathrm{RB}$ decreases dramatically when $50 \mathrm{mg}$ of the flower-like $\alpha-\mathrm{Fe}_{2} \mathrm{O}_{3}$ sample is added and the total degradation of $\mathrm{RB}$ is completed within 35 min (Fig. S3 in the ESM). The superior photocatalytic activity of the flower-like $\alpha-\mathrm{Fe}_{2} \mathrm{O}_{3}$ in comparison to the commercial hematite could be attributed to its high surface area. It is generally accepted that the catalytic process is mainly related to the adsorption and desorption of molecules on the surface of the catalyst. The high specific surface area of the catalyst could result in more unsaturated surface coordination sites exposed to the reactant molecules for catalytic reactions.

\section{Conclusions}

In summary, flower-like $\alpha-\mathrm{Fe}_{2} \mathrm{O}_{3}$ microstructure has been successfully fabricated by simple hydrothermal method following by thermal calcination in air. FESEM and TEM characterizations confirm that the flower-like $\alpha-\mathrm{Fe}_{2} \mathrm{O}_{3}$ microstructure is composed of self-assembled nanorods of about $100 \mathrm{~nm}$ in diameter. The prepared flower-like microstructure shows high photocatalytic activity for the degradation of RB under UV irradiation. This method may present a simple approach for the controlled synthesis of other 3D structures.

\section{Acknowledgements}

This work was financially supported by NRF research grant (No. 3348-20120033).

Electronic Supplementary Material: Supplementary materials are available in the online version of this article at http://dx.doi.org/10.1007/s40145-015-0133-5.

Open Access: This article is distributed under the terms of the Creative Commons Attribution License which permits any use, distribution, and reproduction in any medium, provided the original author(s) and the source are credited.

\section{References}

[1] Lin Y, Yuan G, Sheehan S, et al. Hematite-based solar water splitting: Challenges and opportunities. Energy Environ Sci 2011, 4: 4862-4869.

[2] Wheeler DA, Wang G, Ling Y, et al. Nanostructured hematite: Synthesis, characterization, charge carrier dynamics, and photoelectrochemical properties. Energy Environ Sci 2012, 5: 6682-6702.

[3] Han Y, Wang Y, Li L, et al. Preparation and electrochemical performance of flower-like hematite for lithium-ion batteries. Electrochim Acta 2011, 56: 3175-3181.

[4] Huang X, Guan J, Xiao Z, et al. Flower-like porous hematite nanoarchitectures achieved by complexationmediated oxidation-hydrolysis reaction. $J$ Colloid Interface Sci 2011, 357: 36-45.

[5] Yang Y, Ma H, Zhuang J, et al. Morphology-controlled synthesis of hematite nanocrystals and their facet effects on gas-sensing properties. Inorg Chem 2011, 50: 10143-10151

[6] Wang R, Chen Y, Fu Y, et al. Bicrystalline hematite nanowires. J Phys Chem B 2005, 109: 12245-12249.

[7] Zhang Z, Hossain MF, Takahashi T. Self-assembled hematite $\left(\alpha-\mathrm{Fe}_{2} \mathrm{O}_{3}\right)$ nanotube arrays for photoelectrocatalytic degradation of azo dye under simulated solar light irradiation. Appl Catal B: Environ 2010, 95: 423-429.

[8] Atabaev TS, Vu HHT, Piao Z, et al. Tailoring the luminescent properties of $\mathrm{Gd}_{2} \mathrm{O}_{3}: \mathrm{Tb}^{3+}$ phosphor particles by codoping with $\mathrm{Al}^{3+}$ ions. $J$ Alloys Compd 2012, 541: 263-268.

[9] Cheng X-L, Jiang J-S, Hu M, et al. Controlled synthesis of novel flowerlike $\alpha-\mathrm{Fe}_{2} \mathrm{O}_{3}$ nanostructures via a one-step biphasic interfacial reaction route. Cryst Eng Comm 2012, 14: 7701-7708. 